\title{
A Digital Calibration Technique for Charge Pumps in Phase-Locked Systems
}

\author{
Che-Fu Liang, Student Member, IEEE, Shin-Hua Chen, Student Member, IEEE, and \\ Shen-Iuan Liu, Senior Member, IEEE
}

\begin{abstract}
A digital technique is adopted to calibrate the current mismatch of the charge pump (CP) in phase-locked systems. In this digital calibration technique, there is no extra replica $\mathrm{CP}$ needed. In addition, it can calibrate the $C P$ under different control voltages on the loop filter to be immune to the channel-length modulation. Due to the digital nature, the additional power consumption and digital switching noise from the calibration circuits are turned off once the calibration is finished. A $5 \mathrm{GHz}$ frequency synthesizer is used to justify the proposed calibration technique. The measured output spur is suppressed by $5.35 \mathrm{~dB}$ at $5.2 \mathrm{GHz}$ after the calibration circuits are active. The measured output spur levels are less than $-68.5 \mathrm{dBc}$ throughout the whole output frequency range. The measured phase noise is $-110 \mathrm{dBc} / \mathrm{Hz}$ at an offset frequency of $1 \mathrm{MHz}$.
\end{abstract}

Index Terms-Charge pump (CP) calibration, digital calibration, frequency synthesizer, phase-locked loop (PLL).

\section{INTRODUCTION}

C HARGE pumps (CPs) are widely used in analog phaselocked systems, such as phase-locked loops (PLLs), delay-locked loops (DLLs), and clock and data recovery (CDR) circuits. The $\mathrm{CP}$ converts the phase difference to a proportional voltage to adjust the voltage-controlled oscillator (VCO) or voltage-controlled delay line (VCDL). However, the mismatch between the up and down currents in a conventional CP introduces several problems in phase-locked systems, such as reference spur [1]-[5], static phase error, and jitter. For the short-channel devices in advanced CMOS technology, the device mismatch and channel-length modulation becomes worse. In frequency synthesizers, the mismatch in a CP results in unwanted spurs and deteriorates the spectral performance [1]. In multiphase DLLs [6]-[8], the unbalanced CP creates the static phase error to generate the fixed pattern jitter or degrade the timing margin. Similarly, in conventional CDR circuits, the mismatch in a CP [9] makes the sampling clock away from the optimal position to increase the bit error rate.

Several methods [10]-[12] have been presented to improve the $\mathrm{CP}$ mismatch. In [10], a replica $\mathrm{CP}$ and a digital controller are used to calibrate the $\mathrm{CP}$ mismatch. However, the mismatch between the two CPs still exists. Since two CPs are calibrated on different controlled voltages, it is hard to compensate the current mismatch due to the channel-length modulation. In [11]

Manuscript received November 14, 2006; revised September 28, 2007. This work was supported in part by MediaTek Inc. and by the National Science Council, Taiwan, R.O.C.

The authors are with the Graduate Institute of Electronics Engineering and Department of Electrical Engineering, National Taiwan University, Taipei, Taiwan 10617, R.O.C. (e-mail: 1si@cc.ee.ntu.edu.tw).

Digital Object Identifier 10.1109/JSSC.2007.914283
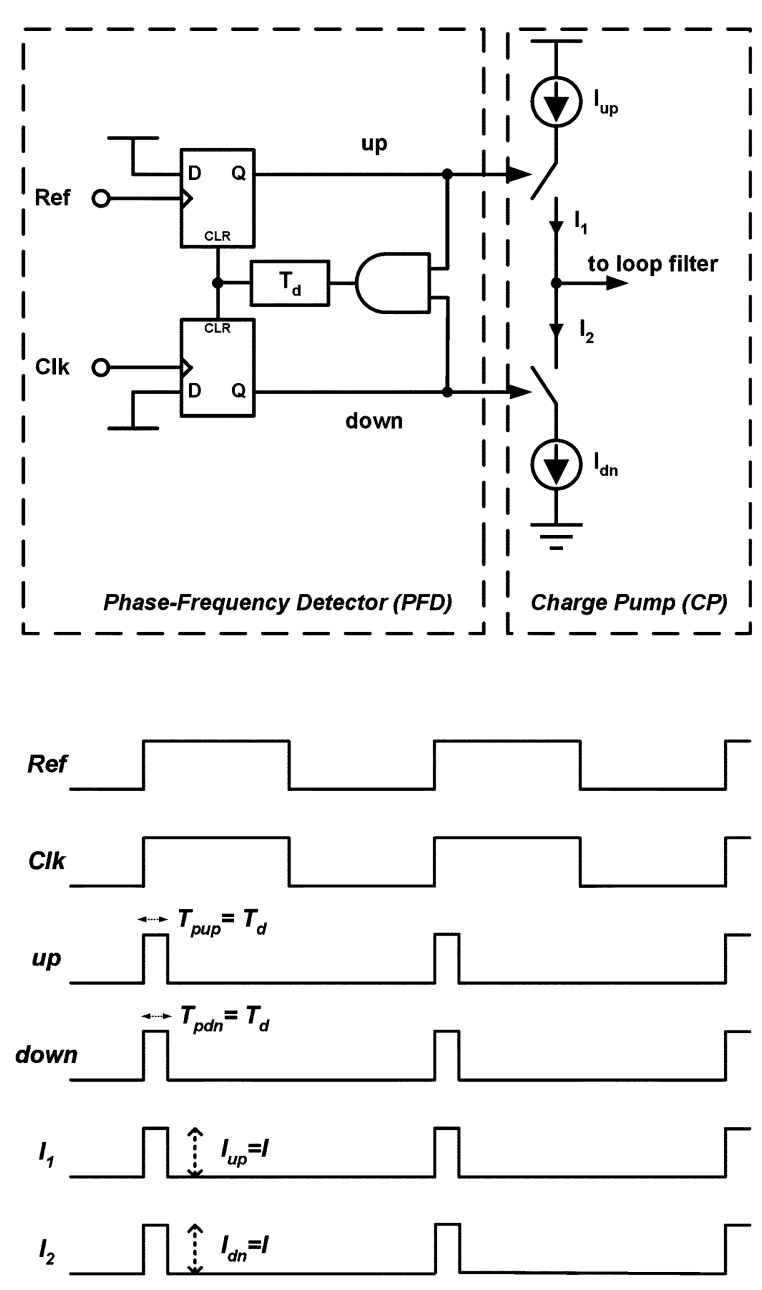

Fig. 1. Traditional PFD and CP.

and [12], the common-mode feedback circuit is used. However, an additional reference voltage and the operational amplifier are needed. The nonideal effects of the operational amplifier have to be considered; such as the stability and offset voltage.

In this paper, a digital calibration technique is presented to solve these problems. It is realized without an additional replica $\mathrm{CP}$. The power consumption and digital switching noise from the calibration circuit are turned off once the calibration is finished. This technique increases the settling time a little. This paper is organized as follows. Section II introduces the digital CP calibration technique. The system architecture, building blocks, and design considerations are presented in Section III. The experimental results are presented in Section IV and a conclusion is presented in Section V. 


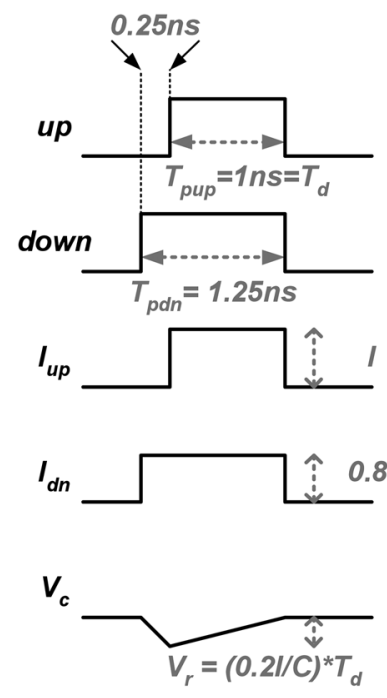

(a)
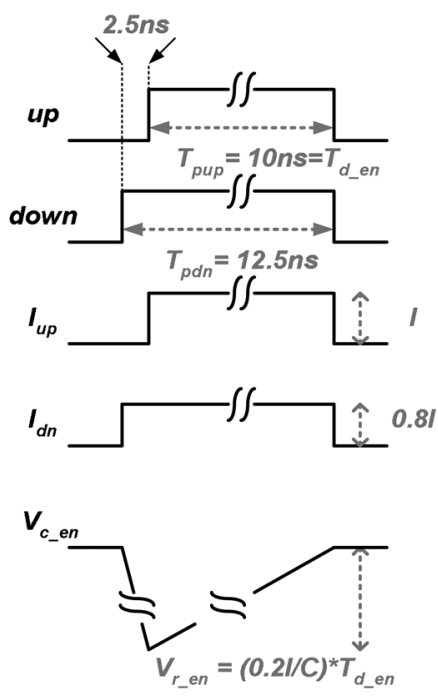

(b)
Fig. 2. Static phase error, current mismatch, and control line ripple: (a) for a normal reset delay and (b) for a enlarged reset delay.

\section{Digital Calibration TechniQue}

Fig. 1 shows a conventional phase-frequency detector (PFD) and a CP. This PFD consists of two resetable D flip-flops (DFFs) and an AND gate. Assuming all the digital gates are ideal, we may model their propagation delays in the reset path of a PFD into a reset delay $T_{d}$, which is appropriately designed to avoid the dead zone. In Fig. 1, the PFD generates two signals, up and down, to control the CP. Assume that the PFD and CP in Fig. 1 are locked in a PLL. Their timing diagrams are shown in Fig. 1 where the high-level pulsewidths are $T_{p \text { up }}$ and $T_{p \text { dn }}$ for the up and down controlling signals, respectively. The current pulses corresponding for the up and down signals are also generated, respectively. Assume these two current pulses are perfectly matched, i.e., $I_{\text {up }}=I_{\text {dn }}=I$ and $T_{p \text { up }}=T_{p \text { dn }}=T_{d}$. When the PLL is locked, the voltage on the loop filter is fixed since the net charge provided by the CP should be zero. To maintain the locking condition, the following equation is always satisfied:

$$
I_{\text {up }} \cdot T_{p \text { up }}=I_{\mathrm{dn}} \cdot T_{p \mathrm{dn}} \cdot
$$

When the CP mismatch exists, it is hard to detect the current mismatch directly. Hence, we have to find a signal, which may represent the amount of this current mismatch. For example, assume that the down current is only $80 \%$ of the up current, i.e., $I_{\mathrm{dn}}=0.8 \cdot I_{\text {up }}$ in Fig. 2(a). Since the up and down currents are not identical, the high-level pulsewidth of the up and down controlling signals should differ as well to satisfy (1). To consider Fig. 2(a), one may derive $T_{p \mathrm{dn}}=1.25 \cdot T_{p \text { up }}=1.25 \cdot T_{d}$ and $I_{\mathrm{dn}}=0.8 \cdot I_{\mathrm{up}}=0.8 \cdot I$. Since both the falling edges of the up and down controlling signals are aligned by the PFD's resetting nature, the rising edge of the down controlling signal leads that of the up controlling signal; i.e., the static phase error exists. Assuming the rest delay $T_{d}=1 \mathrm{~ns}$ in a PFD of Fig. 2(a), it results in the static phase error of $0.25 \mathrm{~ns}$. Similarly, if $I_{\mathrm{dn}}>I_{\mathrm{up}}$, the rising edge of the up controlling signal leads that of the down controlling signal. It inspires us that a bang-bang phase detector

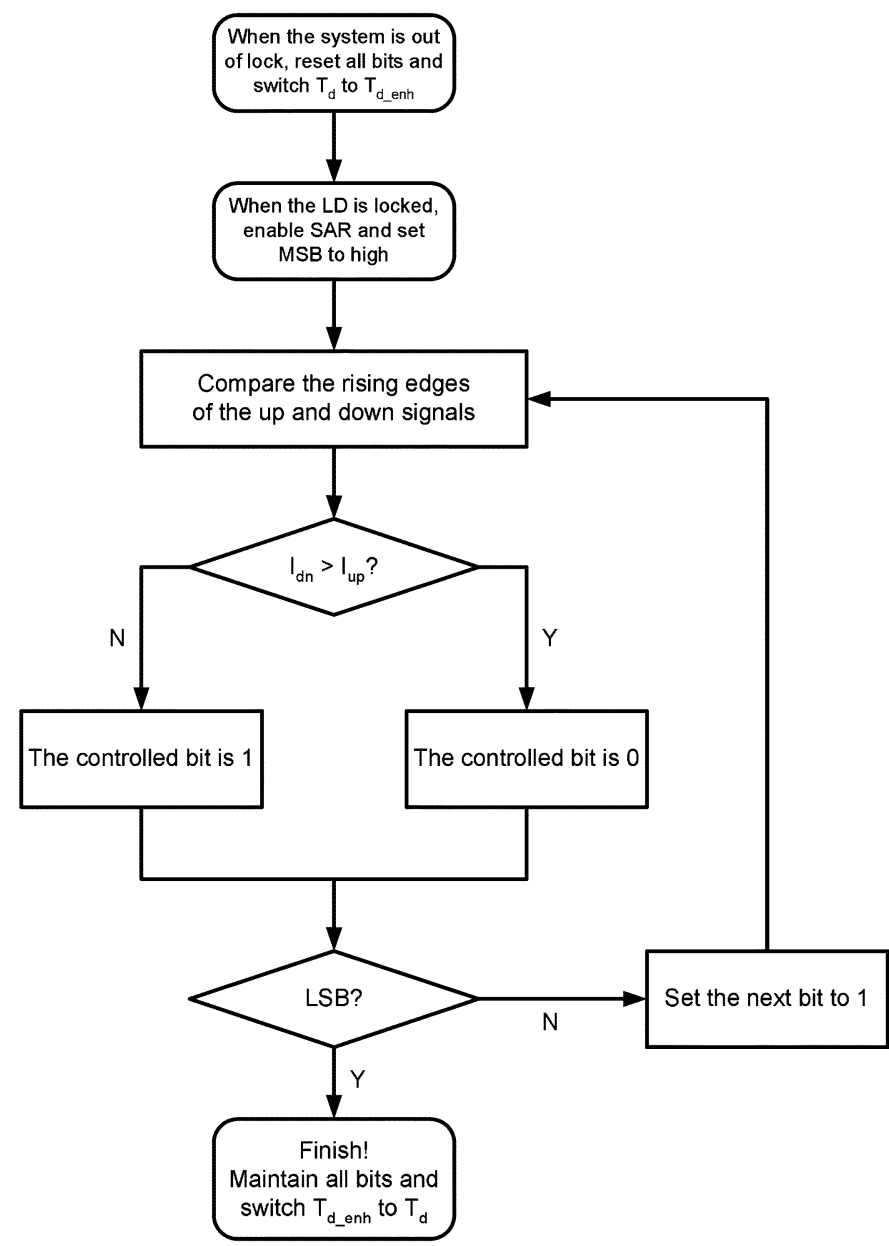

Fig. 3. Flowchart for the digital calibration technique.

(BBPD) can detect this static phase error to know the current mismatch in a $\mathrm{CP}$.

However, this static phase error imposes a hard limitation since it is much smaller than the reset delay $T_{d}$ of a PFD, which is only several nanoseconds. In Fig. 2(a), assume that there is $20 \%$ current mismatch in a CP and $T_{d}$ is $1 \mathrm{~ns}$, the static phase error is only $0.25 \mathrm{~ns}$. To consider a conventional BBPD in a typical $0.18 \mu \mathrm{m}$ CMOS technology, its minimum detectable timing error $\Delta t_{\min }$ is hard to be less than 50 ps. It means that the calibration resolution is only $5 \%$ for the reset delay $T_{d}=1 \mathrm{~ns}$.

In Fig. 2(b), if one can multiply the normal reset delay $T_{d}$ by a factor of 10, the static phase error is also multiplied by the same factor. The calibration resolution in Fig. 2(b) is also enhanced by 10 . The resolution enhancement factor $A_{\text {res }}$ is defined as

$$
A_{\text {res }}=\frac{T_{d_{-\mathrm{en}}}}{T_{d}}
$$

where $T_{d_{-} \text {en }}$ represents the enlarged reset delay of a PFD. However, $T_{d_{-} \text {en }}$ cannot be increased indefinitely. According to the analysis in [13], the maximum reset delay for a PFD should be less than half of the period $T_{\text {ref }}$ of the reference clock to maintain a conventional phase-locked system. Hence, the maximum achievable enhance factor $A_{\text {res_max }}$ is determined as

$$
A_{\text {res_max }}=0.5 \cdot \frac{T_{\text {ref }}}{T_{d}} \text {. }
$$




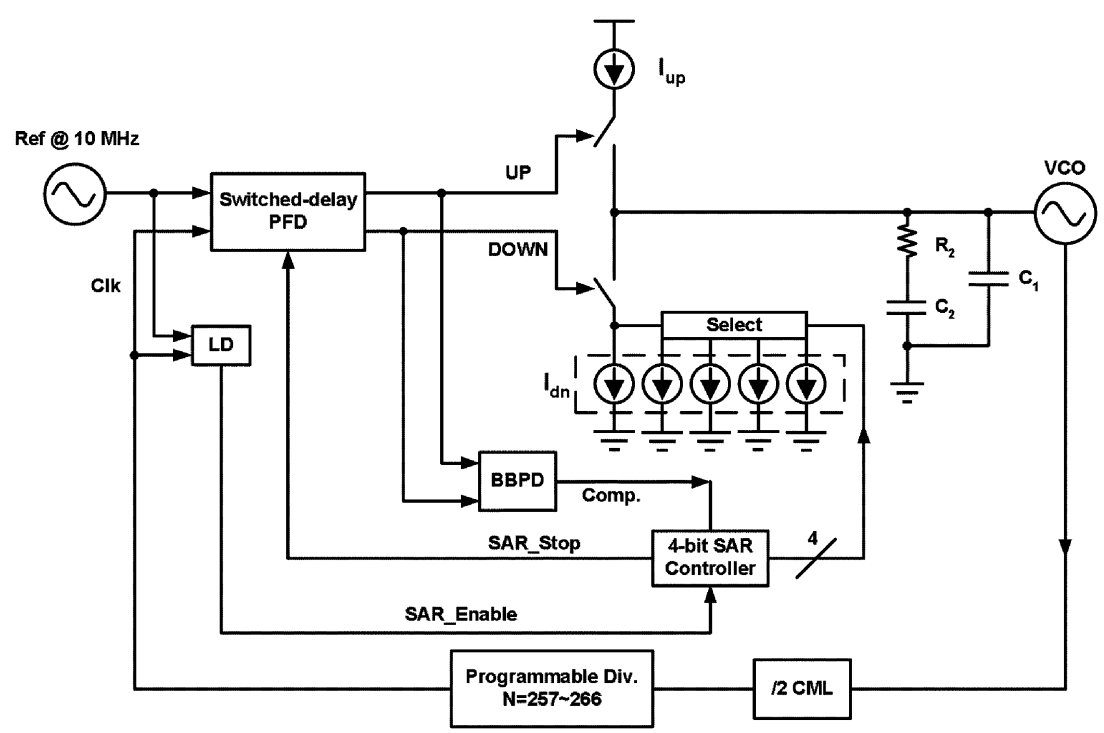

Fig. 4. Frequency synthesizer using the proposed digital calibration technique.

For a conventional BBPD with a minimum detectable timing error $\Delta t_{\text {min }}$, the calibration resolution $R_{\text {cal }}$ is defined as

$$
R_{\text {cal }}=1-\frac{A_{\text {res }} \cdot T_{d}}{\Delta t_{\text {min }}+A_{\text {res }} \cdot T_{d}} .
$$

For example, consider a conventional phase-locked system with a reference clock of $10 \mathrm{MHz}$ and a conventional BBPD with a minimum detectable timing error $\Delta t_{\min }=50 \mathrm{ps}$. According to (2)-(4), the minimum calibration resolution is $0.1 \%$.

Although the calibration resolution can be improved by enlarging the reset delay of a PFD, it is not a good choice for the PFD with the enlarged reset delay in the normal operation. This problem will be discussed as follows. In Fig. 2(a) and (b), the corresponding waveforms for the control voltage $V_{C}$ are plotted, where the loop filter is only the capacitor $C$. The magnitude of the control voltage ripple $V_{r}$ for a normal reset delay $T_{d}$ is given as

$$
V_{r}=\frac{\Delta I}{C} \cdot T_{d}
$$

where $\Delta I$ denotes the current difference between $I_{\mathrm{up}}$ and $I_{\mathrm{dn}}$. In Fig. 2(b), the reset delay of a PFD is multiplied by a factor of 10. Similarly, $V_{r_{-} \text {en }}$ in Fig. 2(b) is ten times larger than that in Fig. 2(a) under the same CP current mismatch. Although $\Delta I$ becomes small after calibration, it is desirable to have a shorter reset delay for a PFD to suppress the control voltage ripple.

Now, the digital calibration technique is described for the $\mathrm{CP}$ in a conventional phase-locked system. Assume the up current is fixed and the down current in the CP is digitally controlled. The successive approximation register-controlled (SAR) scheme [14] is used to have a compromise between calibration time and hardware complexity. The flowchart for the digital calibration technique is shown in Fig. 3. Initially, when the phase-locked system is out of lock, all control bits of the SAR controller are reset. Meanwhile, the reset delay of a PFD is switched to the enlarged one $T_{d-\text { en }}$. When the lock detector (LD) is locked, the CP is calibrated according to the relative phase relationship between the up and down controlling signals of the PFD. The calibration process repeats $N$ rounds for an $N$-bit controlled CP. Finally, all of the control bits are
TABLE I

PARAMETERS FOR THIS SYNTHESIZER

\begin{tabular}{|c|c|}
\hline $\mathrm{C} 1$ & $50 \mathrm{pF}$ \\
\hline $\mathrm{C} 2$ & $1 \mathrm{nF}$ \\
\hline $\mathrm{R} 2$ & $8 \mathrm{kohm}$ \\
\hline Reference Clock & $10 \mathrm{MHz}$ \\
\hline VCO gain & $300 \mathrm{MHz} / \mathrm{v}$ \\
\hline Nominal charge pump current & $200 \mu \mathrm{A}$ \\
\hline Division ratio (N) & $514 \sim 532$ \\
\hline
\end{tabular}

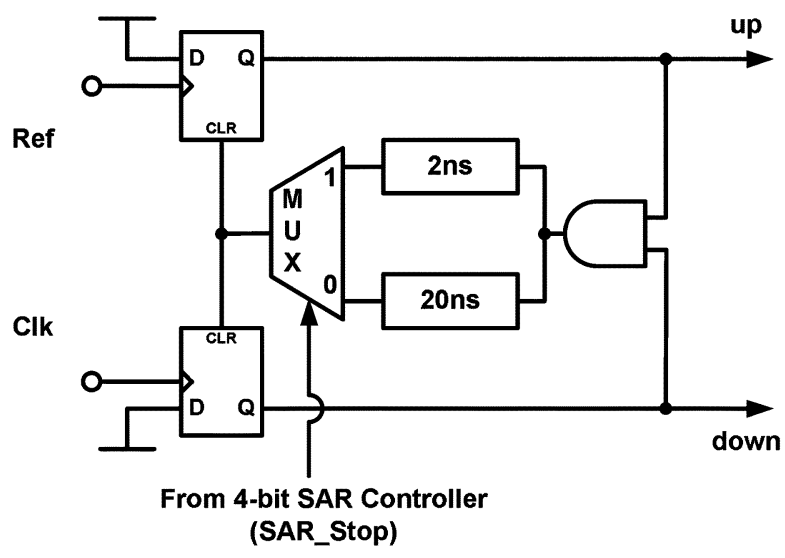

Fig. 5. Switched-delay PFD.

determined and the reset delay of the PFD is switched to the normal one, i.e., $T_{d}$, to further reduce control voltage ripple.

\section{CIRCUIT DESCRIPTION}

To verify the proposed technique, a $5 \mathrm{GHz}$ frequency synthesizer is implemented. The synthesizer aims at the U-NII lower band from 5.18 to $5.32 \mathrm{GHz}$. In Fig. 4, the synthesizer is composed of a traditional integer- $N$ PLL with a switched-delay PFD, a digital-controlled CP, and additional digital calibration circuits. The additional digital calibration circuits include an LD, a BBPD, and a 4-bit SAR controller. The parameters for this synthesizer are listed in Table I. This synthesizer is designed to have a phase margin of $64^{\circ}$ and a loop bandwidth of 


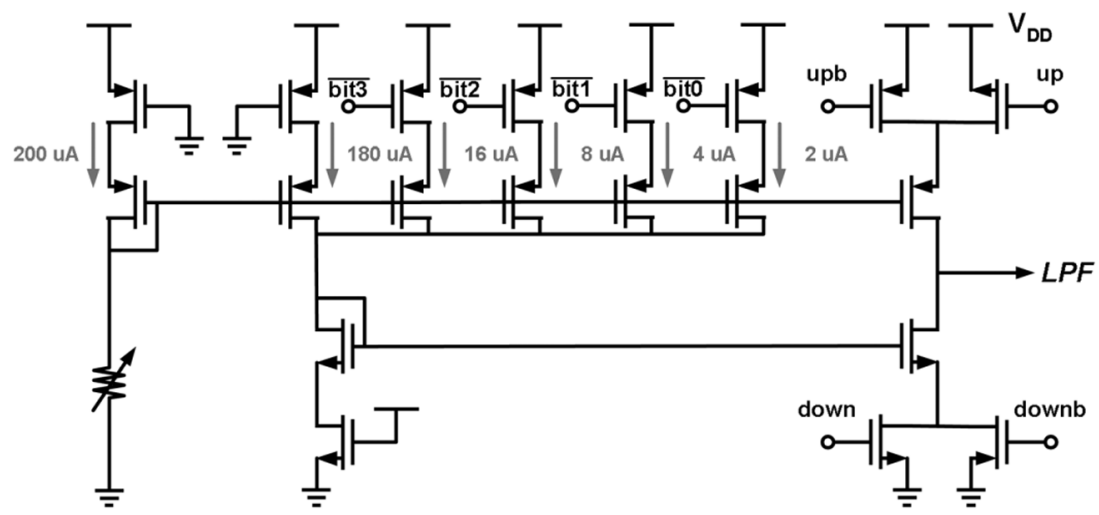

Fig. 6. 4-bit digital-controlled CP.

$200 \mathrm{kHz}$. Once the synthesizer experiences a frequency-hopping command, the LD resets the 4-bit SAR controller to switch the reset delay of a switched-delay PFD to the enlarged one, i.e., $T_{d_{\text {_en }}}$. The 4-bit SAR controller then performs the digital calibration procedure of Fig. 3. Finally, when the calibration is finished, the reset delay of this PFD is switched to the normal one, i.e., $T_{d}$. As a result, the calibration technique is not limited by the channel-length modulation effect under different control voltages and no replica $\mathrm{CP}$ is needed. Furthermore, since the digital calibration circuits are inactive after the calibration is complete, the additional power consumption and digital switching noise from the calibration circuits are turned off. All the building blocks will be illustrated in the following.

\section{A. Switched-Delay PFD}

The switched-delay PFD is shown in Fig. 5 with two reset delays. Two inverter chains are used to realize the normal reset delay of $2 \mathrm{~ns}$ and the enlarged one of $20 \mathrm{~ns}$. Once the LD resets the control bits in the SAR controller, the signal "SAR_stop" will be low to select the enlarged reset delay of $20 \mathrm{~ns}$. When the SAR controller finishes, "SAR_stop" will be high to select the normal reset delay of $2 \mathrm{~ns}$. Based on (4) and $\Delta t_{\min }=50 \mathrm{ps,}$ the calibration resolution of $0.25 \%$ is achieved in our frequency synthesizer.

\section{B. 4-bit Digital-Controlled CP}

To be compliant with the digital calibration technique, the digital-controlled CP is adopted in Fig. 6. The up current has a nominal value of $200 \mu \mathrm{A}$ and changes only with the control voltage on the loop filter due to the channel-length modulation. The down current is digitally controlled and it may vary from 180 to $210 \mu \mathrm{A}$ when the control voltage is pinned at half of the supply voltage, $V_{\mathrm{DD}}=1.8 \mathrm{~V}$. The current step is chosen as $2 \mu \mathrm{A}$ to relieve the worst case current mismatch to $1 \%$ in this digital-controlled CP. In this calibration technique, the control codes of the $\mathrm{CP}$ vary only during the calibration process. After the calibration is complete, the control codes are fixed to avoid extra digital noise. Furthermore, the cascode topology in this 4-bit digital-controlled CP minimizes the digital coupling.

\section{VCO and Dividers}

To have a low sensitivity to the gain of a VCO and a wide tunable frequency range to overcome the process variations, a

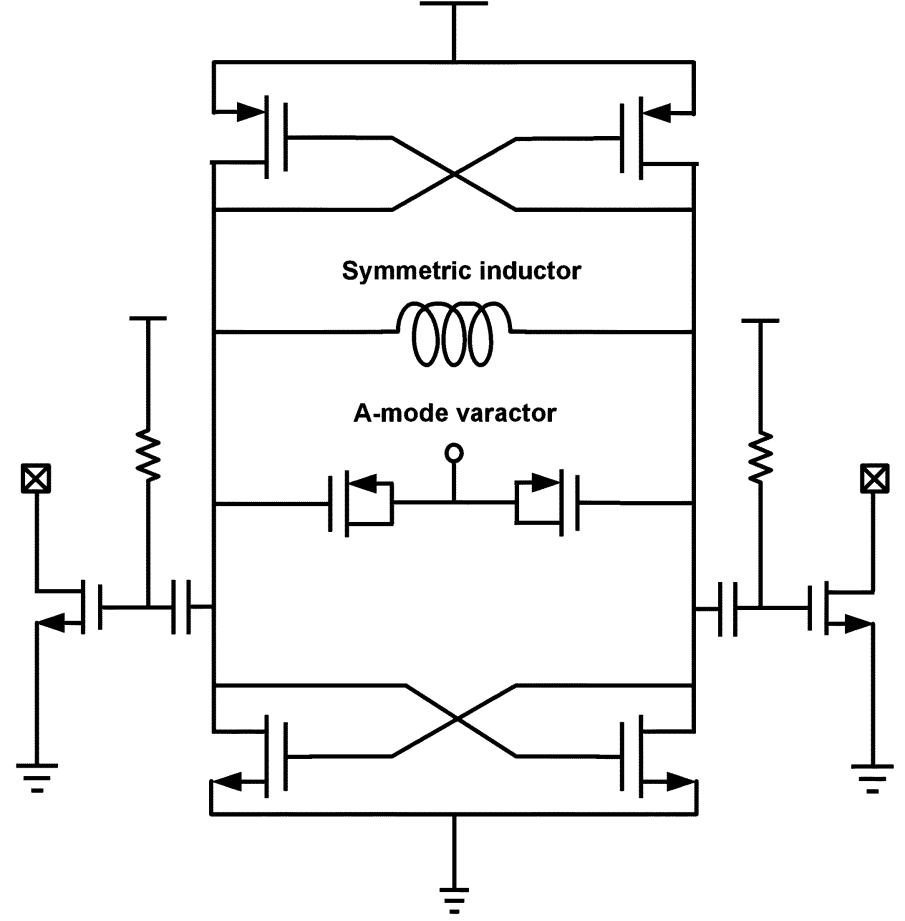

Fig. 7. VCO.

VCO with coarse and fine tunings are usually preferred [2], [3]. However, it needs an additional frequency acquisition circuit. In this work, only one set of the accumulation-mode varactor is used because the calibrated $\mathrm{CP}$ will have a small control voltage ripple. Fig. 7 shows our VCO, which adopts a complementary topology without a tail current source to improve phase noise performance and deliver constant oscillation amplitude [15]. This VCO provides a tuning range from 5 to $5.36 \mathrm{GHz}$ and has a gain of over $300 \mathrm{MHz} / \mathrm{V}$. A symmetric inductor is also used to enhance the phase noise performance. This inductor has a value of $2.2 \mathrm{nH}$ and exhibits a quality factor of 10 at $5 \mathrm{GHz}$. The current consumption of this VCO is $1.5 \mathrm{~mA}$.

The divider chain is composed of a fully differential divideby-two circuit and a multimodulus divider. The divide-by-two circuit is realized by a current-mode logic (CML) DFF [16] to achieve high-speed operation and relax the speed requirement for the following dividers. In our frequency synthesizer, the multimodulus divider in [17] is adopted and it provides a division ratio from 257 to 266 . With a reference clock of $10 \mathrm{MHz}$, 


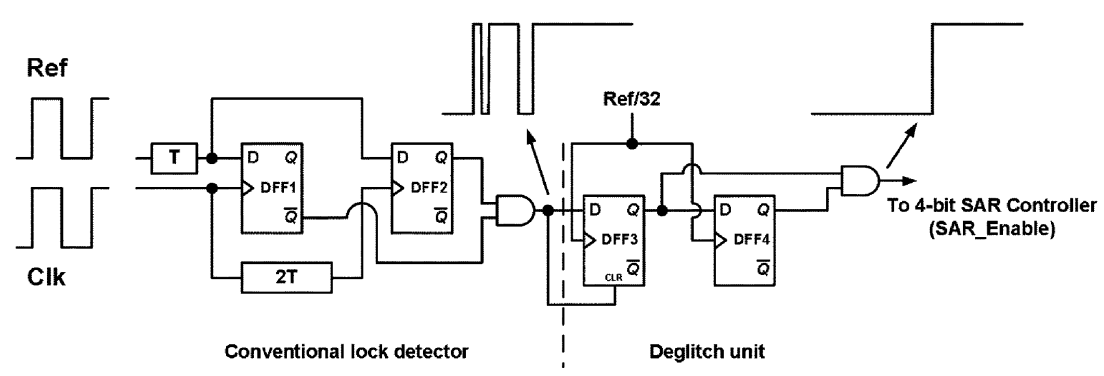

(a)

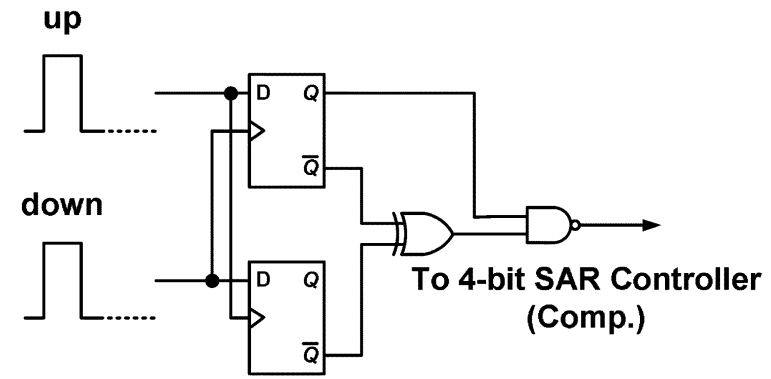

(b)

Fig. 8. (a) LD. (b) BBPD.

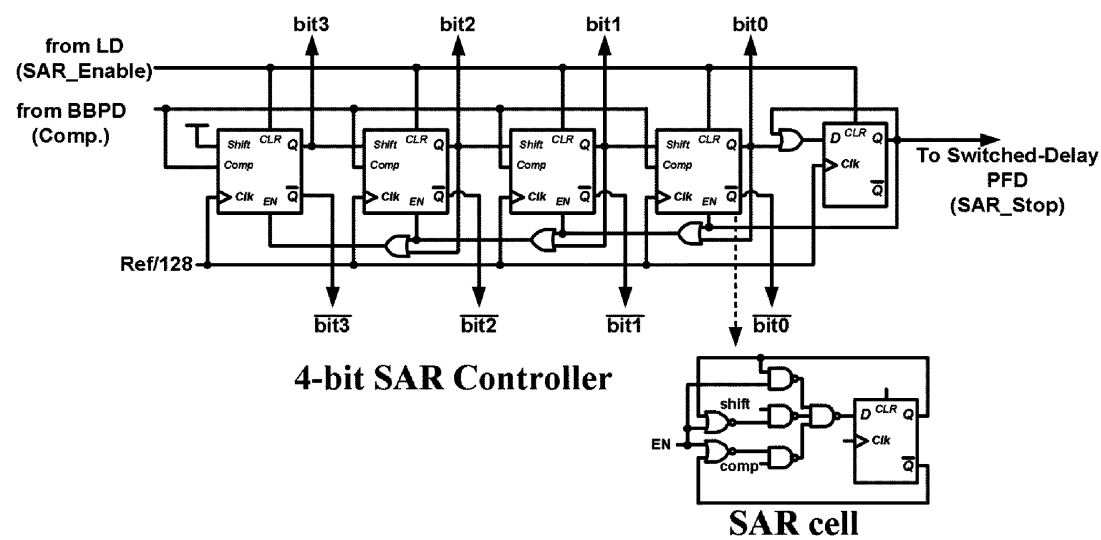

Fig. 9. 4-bit SAR controller.

this frequency synthesizer generates the output frequencies from 5.14 to $5.32 \mathrm{GHz}$ in a step of $20 \mathrm{MHz}$.

\section{D. $L D$ and $B B P D$}

The LD and BBPD are two important circuits in this digital calibration technique. It is because the $\mathrm{LD}$ determines when the digital calibration technique should be reset or turned on. In addition, the BBPD performs the phase comparison between the up and down controlling signals to provide the information for the SAR controller.

The proposed LD is shown in Fig. 8(a) and it is composed of a conventional LD with a window of $\pm T$ (= $4 \mathrm{~ns}$ ) and a deglitch unit. Since the conventional LD has troublesome glitches during the locking transient, the glitches should be eliminated to trigger the digital calibration circuits correctly. The deglitch unit consists of one AND gate and two DFFs (DFF3 and DFF4). These DFFs are triggered by a clock, which is divided by 32 from the input reference clock. During the locking transient, the output of the conventional LD goes high. It is sampled to the output of DFF3 if the divide-by-32 clock is high. If the output of the conventional LD goes low before the next rising edge of the divide-by-32 clock, DFF3 will be reset and the output of the deglitch unit remains low. Conversely, if the output of the conventional LD remains high until the next rising edge of the divide-by-32 clock, both the outputs of DFF3 and DFF4 go high and the proposed LD will output high to indicate a locked condition. The period of the divide-by-32 clock is set to $3.2 \mu \mathrm{s}$, which is long enough to ensure the output of the conventional LD to reach the steady state.

Ideally, a DFF can serve as a simple BBPD to determine the phase relation between the up and down controlling signals. However, in a DFF, there are a finite sampling offset and the unbalanced capacitive load for two controlling signals. To alleviate these problems, the proposed BBPD is shown in Fig. 8(b). The additional DFF not only provides a balanced load for the up and down controlling signals, but also freezes the control codes of the SAR controller when the two controlling signals are very close.

\section{E. 4-bit SAR Controller}

The 4-bit SAR controller in [14] and [18] is adopted as shown in Fig. 9. The operation principle is described as follows. In the beginning, the LD will clear all the cells in the SAR controller. When the first clock arrives, the most significant bit (MSB), i.e., 
bit3, is set to high. The MSB will then be maintained or changed to low according to the comparison result from the BBPD at the next clock edge and the next bit, i.e., bit2, is set to high. Finally, the process will repeat until the least significant bit (LSB), i.e., bit0, is determined. The SAR controller also generates a signal, i.e., "SAR_Stop," to indicate the completeness of the calibration procedure and switch the reset delay of a PFD to the normal one.

The period of the clock in the 4-bit SAR controller is an important parameter. If the clock period is too short, the synthesizer remains unsteady and the BBPD may fail to provide the correct information. Conversely, if the clock period is too long, the total calibration time increases dramatically. As a result, it is necessary to choose an appropriate clock period for our calibration system. Since the $\mathrm{CP}$ is switched during the calibration transient, the synthesizer may experience the phase acquisition. As shown in Fig. 6, the largest current step in the down current is $16 \mu \mathrm{A}$. In the following analysis, the appropriate clock period for our calibration technique is derived.

Assume the synthesizer is locked before the down current is switched and it is modeled as a linear system. A sudden current change $I_{\text {incr }}$ of the down current is modeled as a phase step $\theta_{\text {step }}$ as

$$
\theta_{\text {step }}=\left(\frac{\frac{I_{\mathrm{up}}}{I_{\mathrm{dn} \_ \text {min }}} \cdot T_{d_{-\mathrm{en}}}-\frac{I_{\mathrm{up}}}{I_{\mathrm{dn} \_ \text {min }}+I_{\text {incr }}} \cdot T_{d_{\_ \text {en }}}}{T_{\mathrm{ref}}}\right) \cdot 2 \pi
$$

where $I_{\mathrm{dn} \_m i n}$ denotes the smallest down current in the beginning of the calibration process. Let $I_{\text {up }}, I_{\mathrm{dn} \_ \text {min }}, I_{\text {incr }}, T_{d \_ \text {ehn }}$, and $T_{\text {ref }}$ be 200,180, and $16 \mu \mathrm{A}$, and 20 and $100 \mathrm{~ns}$, respectively. According to (6), the phase step is calculated as $\theta_{\text {step }}=$ $0.114 \mathrm{rad}$ or $6.5^{\circ}$.

The phase transfer function $H(s)$ of the frequency synthesizer on phase domain is shown as

$$
H(s)=\frac{\theta_{\text {out }}(s)}{\theta_{\text {in }}(s)}=\frac{N \cdot \omega_{C} \cdot\left(s+\omega_{Z}\right)}{\frac{s^{3}}{\omega_{P}}+s^{2}+\omega_{C} \cdot s+\omega_{C} \cdot \omega_{Z}}
$$

where

$$
\begin{aligned}
\omega_{P} & =\frac{C_{1}+C_{2}}{C_{1} \cdot C_{2} \cdot R_{2}} \\
\omega_{Z} & =\frac{1}{R_{2} \cdot C_{2}} \\
\omega_{C} & =\frac{I_{\mathrm{cp}} \cdot K_{\mathrm{vco}} \cdot R_{2} \cdot C_{2}}{N \cdot\left(C_{1}+C_{2}\right)}
\end{aligned}
$$

and $I_{\mathrm{cp}}, K_{\mathrm{vco}}$, and $N$ denote the nominal CP current, VCO gain, and division ratio, respectively. To simplify the analysis, the system is designed with the maximum phase margin at the unity gain frequency, i.e., $\gamma \equiv\left(\omega_{C} / \omega_{Z}\right)=\left(\omega_{P} / \omega_{C}\right)$. The phase error transfer function $H_{e}(s)$ between the phase error and input phase is then expressed as

$$
H_{e}(s)=1-\frac{H(s)}{N}=\frac{s^{3}+\omega_{C} \cdot \gamma \cdot s^{2}}{s^{3}+\omega_{C} \cdot \gamma \cdot s^{2}+\omega_{C}^{2} \cdot \gamma \cdot s+\omega_{C}^{3}} .
$$

Finally, the step response of the phase error $\theta_{e_{-} s r}(s)$ can be derived as

$$
\begin{aligned}
\theta_{e_{-} s r}(s) & =\frac{\theta_{\text {step }}}{s} \cdot H_{e}(s) \\
& =\frac{\theta_{\text {step }} \cdot\left(s^{2}+\omega_{c} \cdot \gamma \cdot s\right)}{s^{3}+\omega_{C} \cdot \gamma \cdot s^{2}+\omega_{C}^{2} \cdot \gamma \cdot s+\omega_{C}^{3}} .
\end{aligned}
$$

The stability of the system is heavily related to the value of $\gamma$. In order to have a well-controlled settling behavior, the phase margin of $64^{\circ}$ and $\gamma$ of 4.5 are chosen. It ensures that there is no under-damping settling behavior. If $\gamma>3$, (9) can be further decomposed into (10) as

$$
\theta_{e_{-} s r}(s)=\frac{\theta_{\text {step }} \cdot \frac{(1-\gamma)}{(3-\gamma)}}{s+\alpha_{1}}+\frac{\frac{\theta_{\text {step }}}{(3-\gamma)}}{s+\alpha_{2}}+\frac{\frac{\theta_{\text {step }}}{(3-\gamma)}}{s+\alpha_{3}}
$$

where $\alpha_{1}=\omega_{C}, \alpha_{2}=\left(\left(\gamma-1-\sqrt{\gamma^{2}-2 \gamma-3}\right) / 2\right) \cdot \omega_{C}$, and $\alpha_{3}=\left(\left(\gamma-1+\sqrt{\gamma^{2}-2 \gamma-3}\right) / 2\right) \cdot \omega_{C}$.

We also know that $\alpha_{1}, \alpha_{2}$, and $\alpha_{3}$ are positive real numbers for $\gamma>3$. Now we can derive the step response of the phase error in the time domain

$$
\begin{aligned}
\theta_{e_{-} s r}(t)=\frac{\theta_{\text {step }} \cdot(1-\gamma)}{(3-\gamma)} \cdot e^{-\alpha_{1} \cdot t}+ & \frac{\theta_{\text {step }}}{(3-\gamma)} \cdot e^{-\alpha_{2} \cdot t} \\
& +\frac{\theta_{\text {step }}}{(3-\gamma)} \cdot e^{-\alpha_{3} \cdot t} .
\end{aligned}
$$

Substituting the corresponding values of this synthesizer into (11), the step response of the phase error is shown in Fig. 10(a). Referring to Fig. 6 and (6), the smallest phase step $\theta_{\text {step_min }}$ is $0.015 \mathrm{rad}$ when the system experiences a $2 \mu \mathrm{A}$ down current change in the CP. From Fig. 10(a), a calibration period larger than $4 \mu$ s is enough for a phase error smaller than $\theta_{\text {step_ min }}$ to ensure the calibration resolution.

In this work, assume that the down current is initially less than the up current in the $\mathrm{CP}$. We detect the polarity of the static phase error to increase the down current in the $\mathrm{CP}$ current. The binary-weighted current is updated by the SAR controller. The updated process is from the MSB to LSB to minimize the current mismatch in the CP. The period of the calibration clock for the 4-bit SAR controller is chosen as $12.8 \mu$ s. Furthermore, as described in Section III-D, the switched-delay PFD enables a detecting resolution of $0.25 \%$, which is enough to detect the $1 \%$ current step $(2 \mu \mathrm{A})$ in the CP. As a result, the calibration process may guarantee a minimum current mismatch in the $\mathrm{CP}$.

A behavior simulation using the parameters in Table I is performed and the result is shown in Fig. 10(b). This frequency synthesizer experiences a frequency change from 5.18 to $5.26 \mathrm{GHz}$. It takes about $22 \mu \mathrm{s}$ for the $\mathrm{LD}$ to acquire the lock condition. The calibration procedure spends $51.2 \mu$ s under the calibration period of $12.8 \mu \mathrm{s}$. Finally, the total settling time is about $80 \mu \mathrm{s}$ for this frequency synthesizer to settle within $20 \mathrm{ppm}$ of the target frequency. It should be noted that the calibration procedure is performed once the frequency changes. In fact, the digital codes do not vary much across the full VCO tuning range. To overcome the PVT variations, this technique can be refreshed in the idle time, or the 4-bit SAR controller is executed only once and then transformed into a 4-bit counter [19] to overcome the PVT variations. 


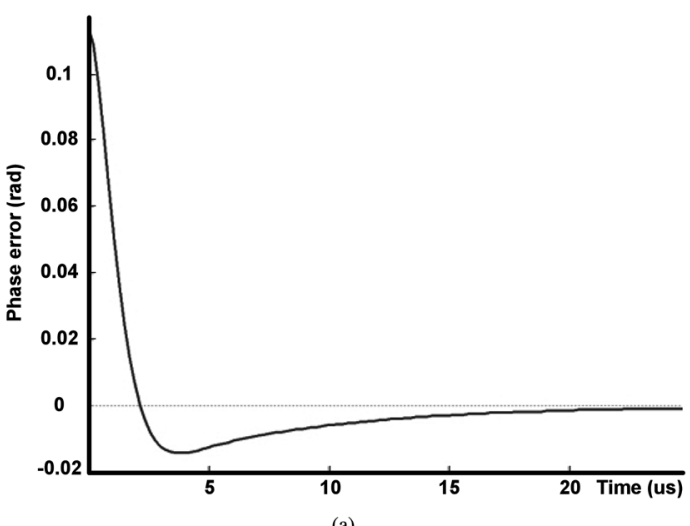

(a)

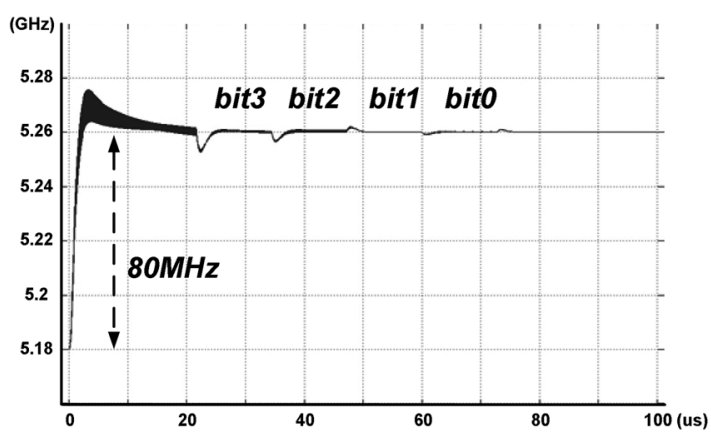

(b)

Fig. 10. (a) Step response for $16 \mu \mathrm{A}$ current change, (b) Behavior simulation from 5.18 to $5.26 \mathrm{GHz}$.

\section{F. Theoretical Spur Level and Nonidealities}

Once the parameters of the PLL are determined, the reference spur can be calculated approximately [20], [21] by assuming a rectangular current pulse and neglecting the PFD's delay time. The reference spur $P_{\text {spur }}$ is given as [20], [21]

$$
\begin{aligned}
P_{\text {spur }}=20 \log \left[\frac{I_{\mathrm{cp}} \cdot R_{2} \cdot K_{\mathrm{vco}} \cdot T_{d}}{\sqrt{2}} \cdot \frac{\Delta I}{I_{\mathrm{cp}}}\right] \\
-20 \log \left(\frac{f_{\mathrm{ref}}}{f_{p 1}}\right)(\mathrm{dBc})
\end{aligned}
$$

where $f_{\text {ref }}$ is the input reference frequency, $I_{\mathrm{cp}}$ is the nominal $\mathrm{CP}$ current, $\Delta I$ is the $\mathrm{CP}$ current mismatch, and $f_{p 1}(=$ $\left.\left(C_{1}+C_{2}\right) /\left(2 \pi \cdot R_{2} \cdot C_{1} \cdot C_{2}\right)\right)$ is the pole of the loop filter. Assume the initial current mismatch is $10 \%$ and the calibrated current mismatch is $1 \%$ for the CP. By using (12) and the parameters in Table I, the calculated reference spur without and with calibration is -51 and $-71 \mathrm{dBc}$, respectively. However, some effects will degrade the reference spur further, such as undesired coupling from the substrate and supply voltage and the reference clock feedthrough from the parasitic capacitor and routed metal lines [22]. The practical improvement may not be as good as expected.

\section{EXPERIMENTAL RESULTS}

This chip has been fabricated in a $0.18 \mu \mathrm{m}$ CMOS $1 \mathrm{P} 6 \mathrm{M}$ mixed-signal technology. A die photograph is shown in Fig. 11. The total chip area and active region occupy $1.4 \mathrm{~mm} \times 1.2 \mathrm{~mm}$ and $0.8 \mathrm{~mm} \times 0.8 \mathrm{~mm}$, respectively. The measured tuning curve of the VCO is shown in Fig. 12. The VCO covers a frequency

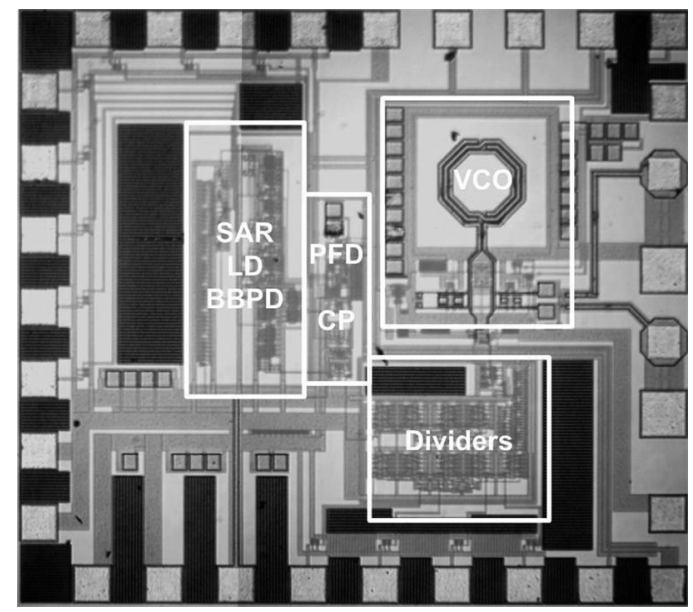

Fig. 11. Die photograph.

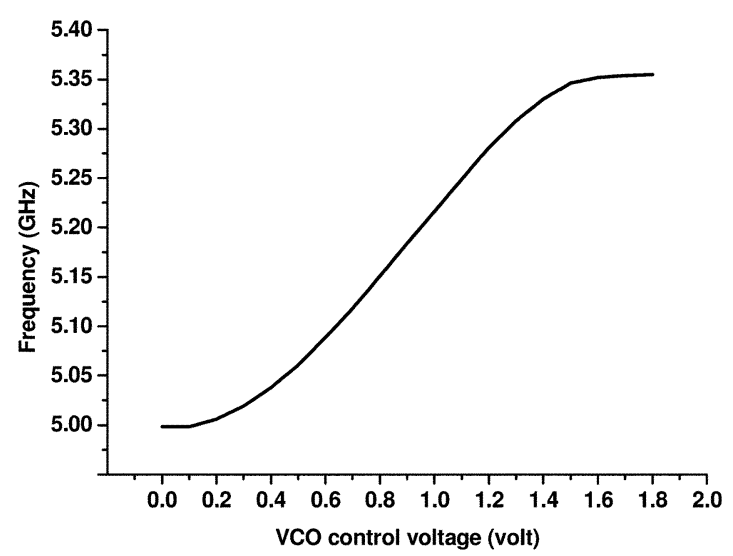

Fig. 12. Measured tuning curve of the VCO.

range from 5 to $5.36 \mathrm{GHz}$ and it is suitable for applications in lower U-NII band (5.15-5.35 GHz). The measured spectral performance without and with the $\mathrm{CP}$ calibration is shown in Fig. 13(a) and (b), respectively. The carrier frequency is chosen to be $5.22 \mathrm{GHz}$ because the VCO has a maximum tuning gain here. The output spurs are further suppressed by $5.35 \mathrm{~dB}$ when the calibration circuits are activated. Fig. 14 shows the measured spurs versus the synthesizer's output frequencies. Our synthesizer has the output spur levels less than $-68.5 \mathrm{dBc}$ throughout the output frequency range. The measured phase noise is $-110 \mathrm{dBc} / \mathrm{Hz}$ at an offset frequency of $1 \mathrm{MHz}$ from the carrier, as shown in Fig. 15.

The digital calibration transient is measured by a modulation analyzer. The frequency hops from 5.18 to $5.26 \mathrm{GHz}$ with an $80 \mathrm{MHz}$ step the same as [2]. Limited by the maximum detectable frequency of $2.5 \mathrm{GHz}$ in this modulation analyzer, the locked synthesizer's output had been down-converted by a $4 \mathrm{GHz}$ tone using a Mini-Circuit ZMX-8 GH mixer in advance of the analyzer's input. The measured switching process in Fig. 16 shows that the synthesizer takes about $80 \mu$ s to settle within $20 \mathrm{ppm}$ of the target frequency, which meets the simulation in Section III.

Table II gives the performance summary of the proposed frequency synthesizer with those in literature. Compared with other works, our synthesizer has a very low $f_{\text {Ref }}$-bandwidth 


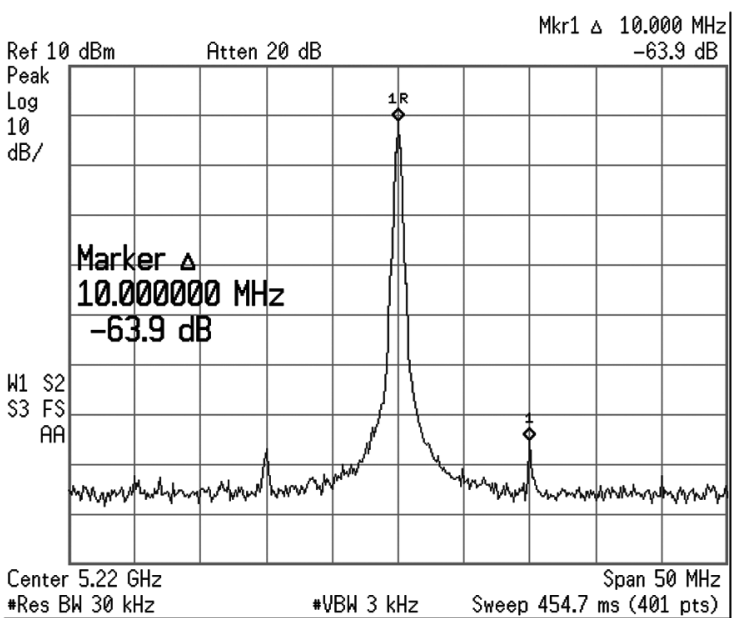

(a)

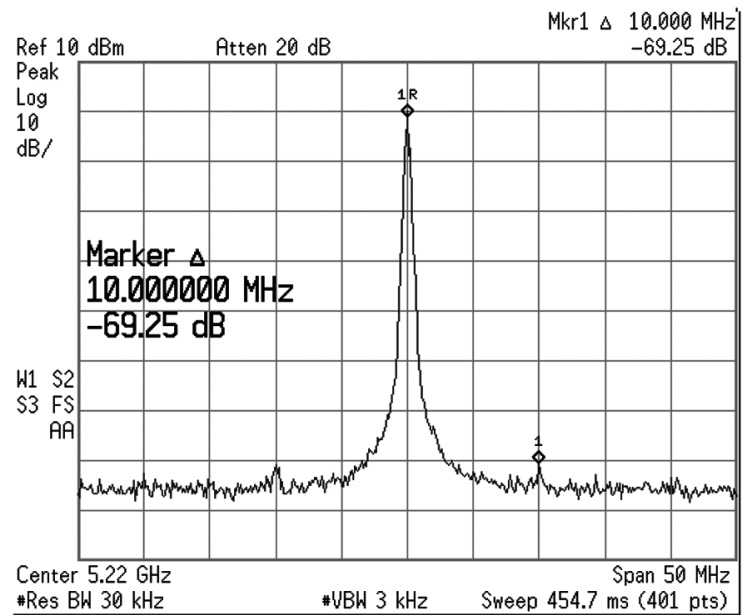

(b)

Fig. 13. Synthesizer spectrum: (a) without calibration and (b) with calibration.

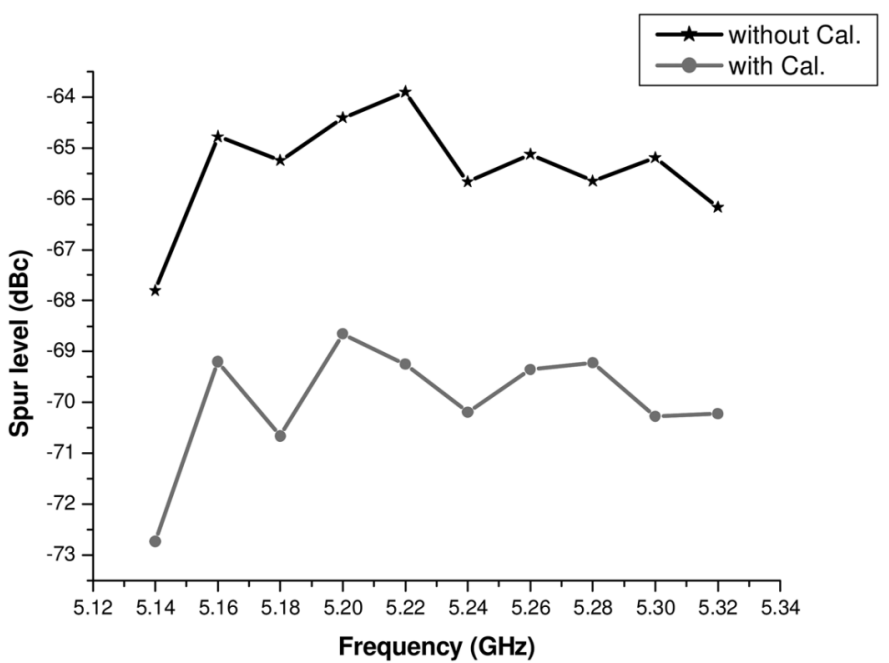

Fig. 14. Measured spur levels versus output frequency.

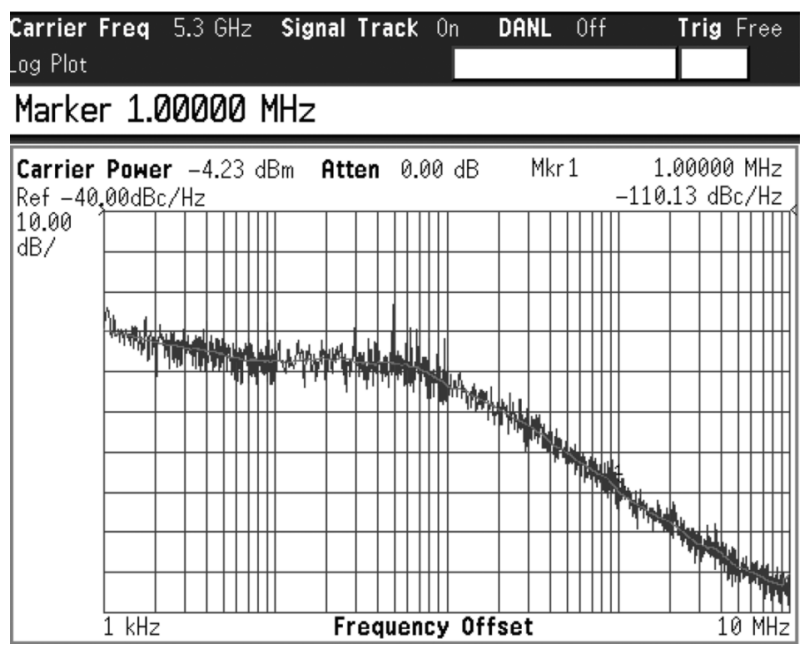

Fig. 15. Measured synthesizer phase noise.

ratio, but still presents a comparable spur level and frequency switching time. Furthermore, only a second-order loop filter is used in this work.

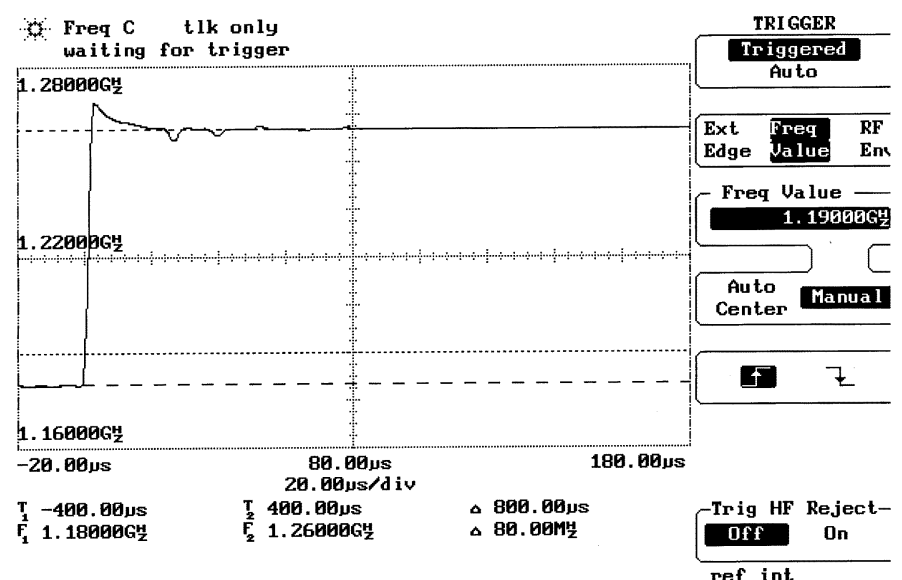

Fig. 16. Measured frequency switching behavior from 5.18 to $5.26 \mathrm{GHz}$.

TABLE II

SUMMARY AND COMPARISON

\begin{tabular}{|c|c|c|c|c|c|}
\hline Ref. & {$[2]$} & {$[3]$} & {$[4]$} & {$[5]$} & This work \\
\hline Freq. $(\mathrm{GHz})$ & $5.14-5.7$ & $4.12-4.72$ & $5.15-5.825$ & $5.15-5.35$ & $5.14-5.32$ \\
\hline Tech. & $\begin{array}{c}0.25 \mu \mathrm{m} \\
\text { CMOS }\end{array}$ & $\begin{array}{c}0.25 \mu \mathrm{m} \\
\text { CMOS }\end{array}$ & $\begin{array}{c}0.18 \mu \mathrm{m} \\
\text { CMOS }\end{array}$ & $\begin{array}{c}0.18 \mu \mathrm{m} \\
\text { CMOS }\end{array}$ & $\begin{array}{c}0.18 \mu \mathrm{m} \\
\text { CMOS }\end{array}$ \\
\hline $\mathrm{f}_{\text {Ref }}(\mathrm{MHz})$ & 10 & 4 & 10 & 13.3 & 10 \\
\hline $\mathrm{BW}(\mathrm{kHz})$ & 25 & 90 & 150 & 200 & 200 \\
\hline $\begin{array}{c}\text { Settling time } \\
(\mu \mathrm{s})\end{array}$ & 100 & $<60$ & N/A & N/A & 80 \\
\hline $\begin{array}{c}\text { Phase noise } \\
\text { @ 1MHz off. } \\
(\mathrm{MBc} / \mathrm{Hz})\end{array}$ & -116 & -87 & -115 & -113 & -110 \\
\hline Loop filter & $3 \mathrm{rd}$ & $2 \mathrm{nd}$ & $3 \mathrm{rd}$ & $2 \mathrm{nd}$ & $2 \mathrm{nd}$ \\
\hline Spurs $(\mathrm{dBc})$ & -70 & -45 & $<-65$ & $<-66$ & $<-68.5$ \\
\hline Power(mW) & 13.5 & 117.5 & N/A & 56 & 19.8 \\
\hline
\end{tabular}

\section{CONCLUSION}

A digital calibration technique has been proposed to alleviate the mismatch in conventional CP circuits. Unlike the previous method with a replica $\mathrm{CP}$, this proposed technique calibrates the $\mathrm{CP}$ circuit in the loop without a replica CP. For the wideband applications, the control voltage of the VCO may vary in a wide range. Our technique may provide a good way to compensate the $\mathrm{CP}$ current mismatch due to the channel-length modulation. The technique consumes negligible power with a cost of extra 
calibration time. However, the extra calibration time can be further reduced by transforming the SAR controller into a counter once the SAR controller has completed.

\section{ACKNOWLEDGMENT}

The authors would like to thank the National Chip Implementation Center for chip implementation.

\section{REFERENCES}

[1] B. Razavi, "Challenges in the design of frequency synthesizers for wireless applications," in IEEE Custom Integrated Circuits Conf., May 1997, pp. 395-402.

[2] S. Pellerano, S. Levantino, C. Samori, and A. L. Lacaita, "A 13.5-mW 5-GHz frequency synthesizer with dynamic-Logic frequency divider," IEEE J. Solid-State Circuits, vol. 39, no. 2, pp. 378-383, Feb. 2004.

[3] F. Herzel, G. Fischer, and H. Gustat, "An integrated CMOS RF synthesizer for 802.11a wireless LAN," IEEE J. Solid-State Circuits, vol. 38, no. 10, pp. 1767-1770, Oct. 2003.

[4] I. Bouras, S. Bouras, T. Georgantas, N. Haralabidis, G. Kamoulakos, C. Kapnistis, S. Kavadias, Y. Kokolakis, P. Merakos, J. Rudell, S. Plevridis, I. Vassiliou, K. Vavelidis, and A. Yamanaka, "A digitally calibrated 5.15-5.825 GHz transceiver for 802.11a wireless LANs in 0.18 $\mu \mathrm{m}$ CMOS," in IEEE Int. Solid-State Circuits Conf., Feb. 2003, pp. 352-353.

[5] P. Zhang, T. Nguyen, C. Lam, D. Gambetta, C. Soorapanth, B. Cheng, S. Hart, I. Sever, T. Bourdi, A. Tham, and B. Razavi, "A direct conversion CMOS transceiver for IEEE 802.11a WLANs," in IEEE Int. Solid-State Circuits Conf., Feb. 2003, pp. 354-355.

[6] G. Chien and P. Gray, "A $900 \mathrm{MHz}$ local oscillator using a DLLbased frequency multiplier technique for PCS applications," in IEEE Int. Solid-State Circuits Conf., Feb. 2000, pp. 202-203.

[7] C. Kim, I.-C. Hwang, and S.-M. Kang, "A low-power small-area \pm 7.28 -ps-jitter 1-GHz DLL-based clock generator," IEEE J. Solid-State Circuits, vol. 37, no. 12, pp. 1414-1420, Dec. 2002.

[8] T.-C. Lee and K.-J. Hsiao, "The design and analysis of a DLL-based frequency synthesizer for UWB application," IEEE J. Solid-State Circuits, vol. 41, no. 6, pp. 1245-1252, Jun. 2006.

[9] B. Razavi, "Challenge in the design of high-speed clock and data recovery circuits," IEEE Commun. Mag., vol. 40, pp. 94-101, Aug. 2002.

[10] H. Huh, Y. Koo, K. Y. Lee, Y. Ok, S. Lee, D. Kwon, J. Lee, J. Park, K. Lee, D. K. Jeong, and W. Kim, "A CMOS dual-band fractional- $N$ synthesizer with reference doubler and compensated charge pump," in IEEE Int. Solid-State Circuits Conf., Feb. 2004, pp. 100-101.

[11] N. D. Dalt and C. Sandner, "A subpicosecond jitter PLL for clock generation in $0.12 \mu \mathrm{m}$ digital CMOS," IEEE J. Solid-State Circuits, vol. 38, no. 7, pp. 1275-1278, Jul. 2003.

[12] S. Cheng, H. Tong, J. S.-Martinez, and A. I. Karsilayan, "Design and analysis of an ultrahigh-speed glitch-free fully differential charge pump with minimum output current variation and accurate matching," IEEE Trans. Circuits Syst. II, Exp. Briefs, vol. 53, no. 9, pp. 843-847, Sep. 2006.

[13] M. Mansuri, D. Liu, and C.-K. K. Yang, "Fast frequency acquisition phase-frequency detectors for Gsamples/s phase-locked loops," IEEE J. Solid-State Circuits, vol. 37, no. 10, pp. 1331-1334, Oct. 2002.

[14] A. Rossi and G. Fucilli, "Nonredundant successive approximation register for A/D converters," Electron. Lett., vol. 32, no. 12, pp. 1055-1057, Jun. 1996.

[15] S. Laventino, C. Samori, A. Bonfanti, A. Lacaita, and V. Boccuzzi, "Frequency dependence on bias current in 5 GHz CMOS VCOs: Impact on tuning range and flicker noise upconversion," IEEE J. SolidState Circuits, vol. 37, no. 8, pp. 1003-1011, Aug. 2002.

[16] J. E. Rogers and J. R. Long, "A 10-Gb/s CDR/DEMUX with $L C$ delay line VCO in 0.18- $\mu \mathrm{m}$ CMOS," IEEE J. Solid-State Circuits, vol. 37, no. 12, pp. 1781-1789, Dec. 2002.

[17] C. Lam and B. Razavi, "A 2.6-GHz/5.2-GHz frequency synthesizer in 0.4- $\mu \mathrm{m}$ CMOS technology," IEEE J. Solid-State Circuits, vol. 35, no. 5, pp. 788-794, May 2000.
[18] G. K. Dehng, J. M. Hsu, C. Y. Yang, and S. I. Liu, "Clock-deskew buffer using a SAR-controlled delay-locked loop," IEEE J. Solid-State Circuits, vol. 35, no. 8, pp. 1128-1136, Aug. 2000.

[19] R. J. Yang and S. I. Liu, "A 40-550 MHz harmonic-free all-digital delay-locked loop using variable SAR algorithm," IEEE J. Solid-State Circuits, vol. 42, no. 2, pp. 361-373, Feb. 2007.

[20] W. Rhee, "Design of high-performance CMOS charge pumps in phaselocked loops," in IEEE Int. Circuits Syst. Symp., Jun. 1999, vol. 2, pp. 545-548.

[21] C. T. Charles and D. J. Allstot, "A calibrated phase/frequency detector for reference spur reduction in charge-pump PLLs," IEEE Trans. Circuits Syst. II, Exp. Briefs, vol. 53, pp. 822-826, Sep. 2006.

[22] G. Van der Plas, C. Soens, M. Badaroglu, P. Wambacq, and S. Donnay, "Modeling and experimental verification of substrate coupling and isolation techniques in mixed-signal ICs on a lightly-doped substrate," in IEEE VLSI Circuits Symp., Jun. 2005, pp. 280-283.

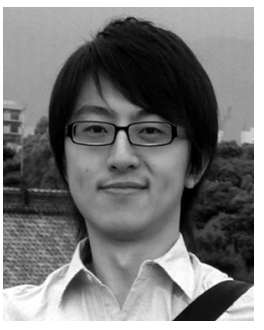

Che-Fu Liang ( $\left.S^{\prime} 04\right)$ was born in Taipei, Taiwan, R.O.C., in 1981. He received the B.S. and Ph.D. degrees in electrical engineering from National Taiwan University, Taipei, Taiwan, R.O.C., in 2003 and 2007, respectively.

His research interests include PLLs and high-speed CMOS data-communication circuits for multiple gigabit applications.

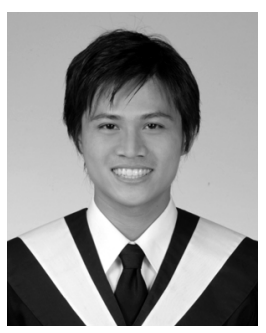

Shin-Hua Chen (S'04) was born in Taipei, Taiwan, R.O.C., in 1982. He received the B.S. and M.S. degrees in electrical engineering from National Taiwan University, Taipei, Taiwan, R.O.C., in 2004 and 2006, respectively.

He is currently with MediaTek Inc., Hsinchu, Taiwan, R.O.C., where he is involved with RF circuit design. His research interests include frequency synthesizers and VCOs.

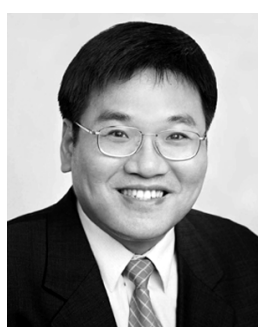

Shen-Iuan Liu (S'88-M'93-SM'03) was born in Keelung, Taiwan, R.O.C., in 1965. He received the B.S. and Ph.D. degrees in electrical engineering from National Taiwan University (NTU), Taipei, in 1987 and 1991, respectively.

During 1991-1993, he served as a second lieutenant in the Chinese Air Force. During 1991-1994, he was an Associate Professor with the Department of Electronic Engineering, National Taiwan Institute of Technology. In 1994, he joined the Department of Electrical Engineering, NTU, where he has been a Professor since 1998. His research interests are analog and digital integrated circuits and systems.

Dr. Liu has served a chair on IEEE SSCS Taipei Chapter since 2004. He has served a general chair on the 15th VLSI Design/CAD Symposium, Taiwan, 2004, and a Program Co-chair on the Fourth IEEE Asia-Pacific Conference on Advanced System Integrated Circuits, Japan, 2004. He was the recipient of the Engineering Paper Award from the Chinese Institute of Engineers, 2003, the Young Professor Teaching Award from MXIC Inc., the Research Achievement Award from NTU, and the Outstanding Research Award from National Science Council, 2004. He has served as a technical program committee member for A-SSCC since 2005 and ISSCC since 2006. He was an Associate Editor for IEEE TRANSACTIONS ON CIRCUITS AND SYSTEMSII: EXPRESS BRIEFS from 2006 to 2007. He has been an Associate Editor for IEEE JOURNAL OF SOLIDStATE Circuits since 2006 and an Associate Editor for IEEE TRANSACTIONS ON CIRCUITS AND SYSTEMSI: REgUlAR PAPERS since 2008. He is a member of the Institute of Electronics, Information and Communication Engineers. 